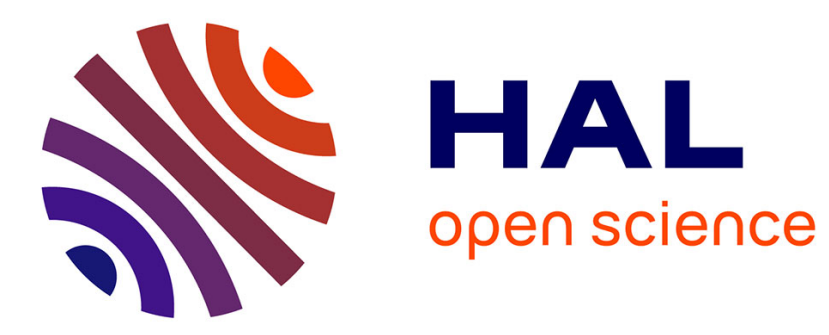

\title{
Reduction method applied to viscoelastically damped finite element models
}

\author{
Souhir Zghal, M.L. Bouazizi, R. Nasri, Noureddine Bouhaddi
}

\section{To cite this version:}

Souhir Zghal, M.L. Bouazizi, R. Nasri, Noureddine Bouhaddi. Reduction method applied to viscoelastically damped finite element models. 4th ECCOMAS Thematic Conference on Computational Methods in Structural Dynamics and Earthquake Engineering (COMPDYN 2013), Jan 2013, Tunisia. pp.1 - 11. hal-00993432

\section{HAL Id: hal-00993432 https://hal.science/hal-00993432}

Submitted on 20 May 2014

HAL is a multi-disciplinary open access archive for the deposit and dissemination of scientific research documents, whether they are published or not. The documents may come from teaching and research institutions in France or abroad, or from public or private research centers.
L'archive ouverte pluridisciplinaire HAL, est destinée au dépôt et à la diffusion de documents scientifiques de niveau recherche, publiés ou non, émanant des établissements d'enseignement et de recherche français ou étrangers, des laboratoires publics ou privés. 


\title{
REDUCTION METHOD APPLIED TO VISCOELASTICALLY DAMPED FINITE ELEMENT MODELS
}

\author{
S. Zghal ${ }^{1,2}$, M-L. Bouazizi ${ }^{2}$, R. Nasri ${ }^{1}$, N. Bouhaddi ${ }^{3}$ \\ ${ }^{1}$ Ecole Nationale d'Ingénieurs de Tunis, Université de Tunis El Manar, Tunisie \\ BP 37,1002 Tunis Belvédère, Tunisie \\ souhirzghal@yahoo.fr; Rachid.Nasri@enit.rnu.tn \\ ${ }^{2}$ Institut Préparatoire aux Etudes d'Ingénieurs de Nabeul, Université de Carthage, Tunisie \\ 8000 M'rezga, Nabeul-Tunisie \\ lamjed.bouazizi@ipein.rnu.tn \\ ${ }^{3}$ Institut FEMTO ST UMR 6174, Département Mécanique Appliquée, Université de Franche-Comté, \\ Besançon-France \\ 24 chemin de l'épitaphe 25000 Besançon-France \\ noureddine.bouhaddi@univ.fcomte.fr
}

Keywords: Sandwich, Viscoelastic Materials, Finite Element Modeling, Guyan condensation, Dynamic Condensation.

\begin{abstract}
We propose in this paper to combine the GHM (Golla-Hughes-Mc Tavish) method with model reduction technique, especially direct condensation methods to resolve the problem of increased model order of viscoelastically structures. In fact, modeling structures using the GHM method leads to global systems of equation of motion whose numbers of degrees-offreedom largely exceeds the order of the associated undamped system. As result, the numerical resolution of such equations can require prohibitive computational (CPU) time. So, to overcome this problem, both Static and Dynamic methods are used to reduce the order of finite elements matrices while preserving its capability to represent the dynamic behavior of viscoelastically damped structures. This paper intends to compare these two methods in direct reduction. Numerical example applied to cantilever beam structure is presented. This example will highlight the domain of validity of the studied methods. Results obtained from these two reduction methods are compared with the full model in order to illustrate its performances and its practical interest in the dynamic analysis of viscoelastically damped structures.
\end{abstract}




\section{INTRODUCTION}

In the design of large industrial structures, the use of viscoelastic sandwich structures [1,2] can provide an effective means of vibration control. However, these structures are highly dependent on the viscoelastic materials properties, which depend strongly on the excitation frequency. Therefore, a correct modeling of the viscoelastic behavior is required for the analysis of such structures. One effective approach used to model the viscoelastic behavior is the Golla-Hughes Mc Tavish [3, 4] model (GHM) which is based on the addition of internal or dissipative variables. This approach leads generally to largely dimension systems. Therefore, a model reduction should be applied to the augmented GHM model.

The present work presents an alternative two reduction methods for this problem. The first one is the Guyan condensation method $[5,6]$ which is applied to the structures through a partition of the equation of motion in term of master and slave coordinates leading to a static transformation whose coordinates are a subset of the original coordinates system. The second reduction is the dynamic condensation method [7] in which some slave modes are retained and added to the Guyan static transformation leading to enriched basis. The reduced order models are compared in terms of accuracy, performance and computational efficiency for the cantilever beam structure.

\section{GHM FINITE ELEMENT MODEL}

The viscoelastic shear modulus is represented, in the GHM model, by a series of functions in the Laplace domain such that:

$$
G(s)=G_{O}\left(1+\sum_{i=1}^{N_{G}} \alpha_{i} \frac{s^{2}+2 \xi_{i} \omega_{i} s}{s^{2}+2 \xi_{i} \omega_{i} s+\omega_{i}^{2}}\right)
$$

Where: $\mathrm{G}_{0}$ is the static modulus; $\mathrm{s}$ is the Laplace complex variable; $\alpha_{i}, \xi_{i}, \omega_{i}$ are the parameters of the i mini-oscillator, and $N_{G}$ is the number of mini-oscillators. This leads to derive the equation of motion in term of structural dofs $\{q\}$ and dissipative dofs $\left\{z_{i}\right\}$ which are defined as follows:

$$
\left\{z_{i}(s)\right\}=\left\{\frac{\omega_{i}^{2}}{s^{2}+2 \xi_{i} \omega_{i} s+\omega_{i}^{2}}\right\}\{q(s)\}
$$

After some manipulations and back to time domain, the following equation of motion in the Laplace domain is obtained:

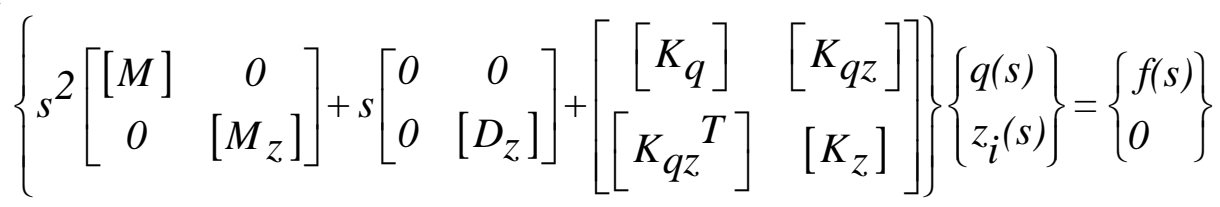

Or in compact form:

$$
\left\{s^{2}\left[M_{G}\right]+s\left[D_{G}\right]+\left[K_{G}\right]\right\}\left\{q_{G}(s)\right\}=\left\{F_{G}(s)\right\}
$$

Where: $\left[M_{G}\right] ;\left[D_{G}\right]$ and $\left[K_{G}\right] \in \mathbb{R}^{t_{G}, t_{G}}$ are respectively the mass, damping and stiffness matrices of viscoelastic GHM model with $t_{G}=N \times\left(1+N_{G}\right)$ and $N$ is the dimension of structural dofs (size of $\{q\}$ ). 


$$
\begin{aligned}
& {\left[M_{G}\right]=\left[\begin{array}{cccc}
{[M]} & 0 & \cdots & 0 \\
0 & \frac{\alpha_{1}}{\omega_{1}^{2}}\left[K_{v}^{0}\right] & 0 & \vdots \\
\vdots & 0 & \ddots & 0 \\
0 & \cdots & 0 & \frac{\alpha_{N} N_{G}}{\omega^{2}}\left[K_{v}^{0}\right.
\end{array}\right] ;\left[D_{G}\right]=\left[\begin{array}{cccc}
0 & 0 & \cdots & 0 \\
0 & \frac{2 \alpha_{1} \xi_{1}}{\omega_{1}}\left[K_{v}^{0}\right] & 0 & \vdots \\
\vdots & 0 & \ddots & 0 \\
0 & \cdots & 0 & \frac{2 \alpha_{N_{G}} \xi_{G}}{\omega_{N_{G}}}\left[K_{v}^{0}\right]
\end{array}\right] ;} \\
& {\left[K_{G}\right]=\left[\begin{array}{cccc}
{\left[K_{e}\right]+\left[K_{v}^{\infty}\right]} & -\alpha_{1}\left[K_{v}^{0}\right] & \ldots & -\alpha_{N_{G}}\left[K_{v}^{0}\right] \\
-\alpha_{1}\left[K_{v}^{0}\right]^{T} & \alpha_{1}\left[K_{v}^{0}\right] & 0 & \vdots \\
\vdots & 0 & \ddots & 0 \\
-{ }_{N_{G}}\left[K_{v}^{0}\right]^{T} & \ldots & 0 & \alpha_{N_{G}}\left[K_{v}^{0}\right]
\end{array}\right] ;\left\{F_{G}\right\}=\left\{\begin{array}{l}
f \\
0
\end{array}\right\} ;\left\{q_{G}\right\}=\left\{\begin{array}{l}
q \\
z_{i}
\end{array}\right\}} \\
& \text { With: }\left[K_{v}^{O}\right]=G_{0}\left[\bar{K}_{v}\right] ;\left[K_{v}^{\infty}\right]=\left[K_{v}^{0}\right]\left(1+\sum_{i=1}^{N_{G}} \alpha_{i}\right) \text { are respectively, the static or }
\end{aligned}
$$

low frequency stiffness matrix and the dynamic or high frequency stiffness matrix.

Consequently, the inclusion of dissipative coordinates increases the order of the differential equation of motion such that the structural degrees-of-freedom are least doubled. This increases the computational time of the system and motivates the use of reduction methods. 


\section{CONDENSATION METHODS}

Models reduction procedures are used in order to reduce the GHM model dimension and his associated large computational times. This can be done based on the assumption that the exact responses given by the resolution of equation (4) can be approached by projections on a reduced vector basis as follows:

$$
\left\{q_{G}\right\}=[T]\left\{q_{C}\right\}
$$

Where: $[T] \in \mathbb{R}^{\mathbf{t}_{\mathbf{G}}, \mathbf{N R}}$ is the transformation matrix formed column wise by a vector basis, $\left\{q_{C}\right\} \in \mathbb{R}^{\mathbf{N R}, \mathbf{1}}$ are the generalized coordinates, and $\mathrm{NR} \ll \mathbf{t}_{\mathbf{G}}$ is the number of reduced vectors in the basis. The generalized coordinates representing the contribution of each column of $[T]$ are chosen arbitrary in which the reduced model provides a reasonable predictive into the frequency bandwidth.

The reduced equation of motion can be written as follows:

$$
\left[M_{c}\right]\left\{\ddot{q}_{c}\right\}+\left[D_{c}\right]\left\{\dot{q}_{c}\right\}+\left[K_{c}\right]\left\{q_{c}\right\}=\left\{f_{c}\right\}
$$

Where: $\left[M_{c}\right] ;\left[D_{c}\right]$ and $\left[K_{c}\right] \boldsymbol{\epsilon} \mathbb{R}^{N R, N R}$ are respectively the reduced mass, stiffness and damping matrices expressed as follows: $\left[M_{C}\right]=[T]^{T}\left[M_{G}\right][T] \quad ; \quad\left[D_{c}\right]=[T]^{T}\left[D_{G}\right][T]$ $\left[K_{c}\right]=[T]^{T}\left[K_{G}\right][T] ;\left\{f_{C}\right\}=[T]^{T}\left\{f_{G}\right\}$

Two reduction methods are adopted regarding the computation of reduction basis: Guyan condensation and Dynamic condensation.

\subsection{Guyan condensation method}

Guyan condensation method $[5,6]$ is based to divide the displacements vector of structural dofs $\{q\}$ into two subvectors:

$\left\{q^{m}\right\} \in \mathbb{R}^{\mathbf{m}, \mathbf{1}}:$ Subvector of master dofs.

$\left\{q^{s}\right\} \in \mathbb{R}^{\mathbf{s}, \mathbf{1}}$ : Subvector of slave dofs.

With $m+s=N$ being the order of the physical dofs of the viscoelastic structure and $\mathrm{m} \ll \boldsymbol{s}$. 
By neglecting the inertia and the external load associated to the first equation of motion (3), partitioning it into master and slave coordinates (or dofs), the reduced model is obtained with the Guyan transformation which can be expressed as: $[T]=\left[\begin{array}{cc}I_{1} & 0 \\ t_{1} & t_{2} \\ 0 & I_{2}\end{array}\right] \boldsymbol{\epsilon} \mathbb{R}^{\boldsymbol{t}_{G}, \boldsymbol{m}+\boldsymbol{N N}_{\boldsymbol{G}}}$

$$
\begin{aligned}
& {\left[t_{1}\right]=-\left[K_{q}^{s S}\right]^{-1}\left[K_{q}^{s m}\right] \epsilon \mathbb{R}^{s, m}} \\
& {\left[t_{2}\right]=-\left[K_{q}^{S S}\right]^{-1}\left[\begin{array}{ll}
K_{q z}^{S m} & K_{q z}^{S S}
\end{array}\right] \boldsymbol{\epsilon} \mathbb{R}^{s, N N_{G}}}
\end{aligned}
$$

In this case, the size of reduced mass, stiffness and damping matrices is $N R=\boldsymbol{m}+\boldsymbol{N} \boldsymbol{N}_{\boldsymbol{G}}$. Consequently, the reduced model have the follows dimension $\left[M_{c}\right] ;\left[D_{c}\right]$ and $\left[K_{C}\right]$ $\epsilon \mathbb{R}^{m+N N_{G}, m+N N_{G}}$.

\subsection{Dynamic condensation method}

This method [7] consists to enrich the Guyan matrix transformation of some slaves modes obtained by the resolution of the slave eigenfrequencies problem as follows:

$$
\left(\left[K_{q}^{s S}\right]-\lambda_{i}\left[M^{s S}\right]\right)\left\{\phi_{i}\right\}=0 \quad i=1, \ldots \ldots, s
$$

Where: $\left[K_{q}^{s s}\right]$ and $\left[M^{s s}\right]$ are respectively the slaves' structural stiffness and mass matrices.

$$
\phi_{i}=\left[\begin{array}{lll}
\phi_{1} & \phi_{2} & \phi_{s}
\end{array}\right] ; \lambda_{i}=\operatorname{diag}\left(\lambda_{1}, \ldots \ldots \ldots, \lambda_{s}\right)
$$

This base is trounced to $\mathrm{p}$ first slave modes $\phi_{s p}=\left[\begin{array}{lll}\phi_{1} & \phi_{2} & \phi_{p}\end{array}\right] \boldsymbol{\epsilon} \mathbb{R}^{\boldsymbol{s}, \boldsymbol{p}}(p \ll \boldsymbol{s})$ which are retained and added to the static basis to enrich it. Thereby, the dynamic transformation can be expressed as follows:

$$
[T]=\left[\begin{array}{ccc}
I_{1} & 0 & 0 \\
t_{1} & t_{2} & \phi_{s p} \\
0 & I_{2} & 0
\end{array}\right] \in \mathbb{R}^{t_{G}, m+N N_{G}+p}
$$

In this case, the size of reduced mass, stiffness and damping matrices is $N R=\boldsymbol{m}+\boldsymbol{N} \boldsymbol{N}_{\boldsymbol{G}}+$ $\boldsymbol{p}$. Consequently, the reduced model have the follows dimension $\left[M_{c}\right] ;\left[D_{c}\right]$ and $\left[K_{C}\right]$ $\epsilon \mathbb{R}^{m+N N_{G}+p, m+N N_{G}+p}$ 


\section{NUMERICAL APPLICATION}

In this section, numerical application is presented to illustrate the main features of the used condensation strategy of viscoelastic sandwich structures. We consider one mini-oscillator $\left(\mathbf{N}_{\mathbf{G}}=1\right)$ of viscoelastic cantilever beam which is constituted by two elastic layers (faces) in Aluminum and a viscoelastic layer (core) of the nuance ISD112. All the calculations are developed using the software MATLAB ${ }^{\circledR}$. The material and geometrical characteristics of the used structure are shown in Table1. The values of the parameters of the viscoelastic commercially available ISD112, manufactured by $3 \mathrm{M}^{\mathrm{TM}}$ used at $27^{\circ} \mathrm{C}$ for one mini-oscillator are presented in Table 2.

\begin{tabular}{|c|c|}
\hline Elastic layers & Shear modulus: $G_{f}=9,6 \times 10^{10} \mathrm{~Pa}$ \\
\hline & Poisson ratio: $v_{f}=0,3$ \\
\hline & Density $: \rho_{\mathrm{f}}=2766 \mathrm{Kg} \cdot \mathrm{m}^{-3}$ \\
\hline & Thickness: $\mathrm{h}_{\mathrm{f}}=1,524 \mathrm{~mm}$ \\
\hline Viscoelastic laver & Shear modulus: GHM modulus (1) \\
\hline (ISD112) & Poisson ratio: $v_{c}=0,49$ \\
\hline & Density $: \rho_{c}=1600 \mathrm{Kg} \cdot \mathrm{m}^{-3}$ \\
\hline & Thickness: $\mathrm{h}_{\mathrm{c}}=0,127 \mathrm{~mm}$ \\
\hline Beam & $\begin{array}{l}\text { Length: } L=177,8 \mathrm{~mm} \\
\text { Width: } 1=12.7 \mathrm{~mm}\end{array}$ \\
\hline
\end{tabular}

Table 1: Material and geometrical properties of the used sandwich structures [8]

\begin{tabular}{ll}
\hline Model GHM (i=1) & Value \\
\hline$\alpha_{i}$ & 4.8278 \\
$\omega_{i}$ & 28045 \\
$\xi_{i}$ & 22.013 \\
$G_{0}[\mathrm{MPa}]$ & 0.1633 \\
\hline
\end{tabular}

Table2. Parameters of the GHM viscoelastic model identified for material ISD112 $3 \mathrm{M}^{\mathrm{TM}}$ for one mini-oscillator 
The used FE mesh of the beam involves one element through the width and 20 elements along the length, containing in total 1000 dofs. The excitation point and the response point are selected at the extremity of the beam as shown in figure 1 .

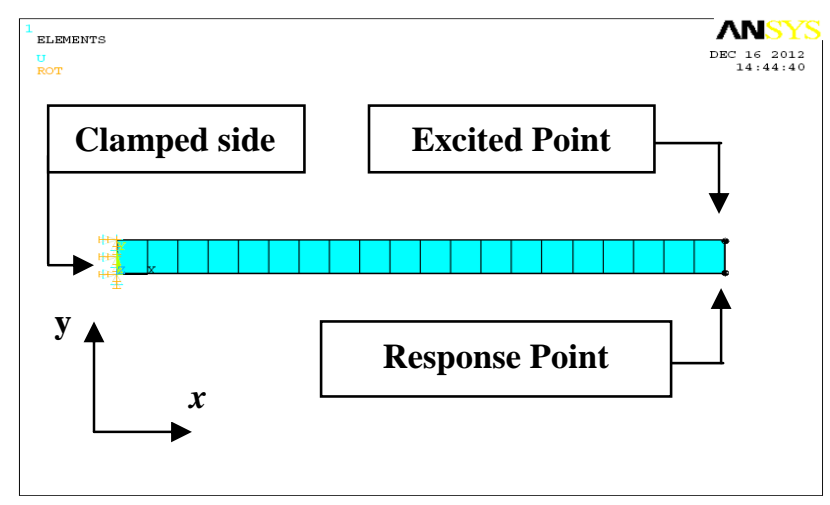

Figure1: Illustration of the FE model implemented for the Clamped-Free (C-F) viscoelastic beam

The response of the damped GHM model (described by rational fraction) and his associated undamped model of the beam under a harmonic excitation are presented in figure 2 .

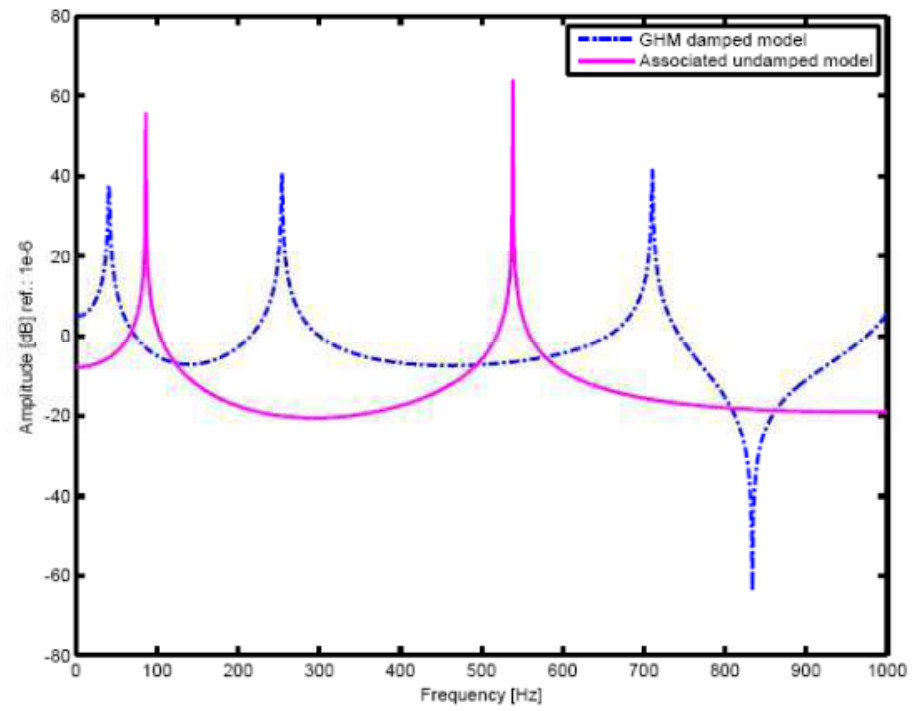

Figure2: FRFs of GHM damped model and his associated undamped model of the viscoelastic sandwich beam

The «blue » curve corresponds to the frequency response of the viscoelastic beam described by the frequency dependent GHM model and the «Magenta » curve is his associated undamped model in the frequency range of [0-1000] Hz. Indeed, these curves show a shift of both amplitudes and frequencies between the GHM rational model and his associated undamped model. This resulting first in dimuniation of amplitudes of $40 \%$ of the viscoelastic damped structure compared to his associated undamped structure.

This is explained by the damping effect introduced by the GHM model form (1) as a series of mini-oscillators while the associated undamped model is described by a static modulus $\mathrm{G}(\omega=0)=\mathrm{G}_{0}$ which does not take account this effect. In second time, the shift of frequencies between the two models allows to determine the natural frequencies (which cannot determine 
them by the classical method such as eigenvalues standard problem or MSE method) and the damped frequencies of the viscoelastic sandwich beam described by rational GHM model.

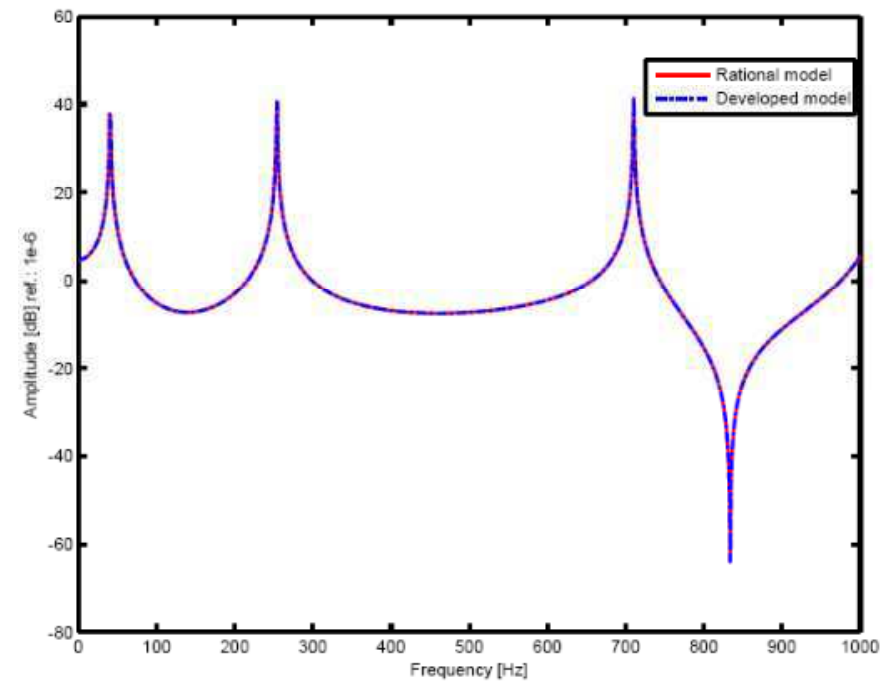

Figure3: FRFs for the rational and the developed GHM model of the viscoelastic sandwich beam

The frequency response of the rational model (1) and the developed model (3) are confused. This leads to conclude that these two models are equivalent. They are used as reference in the modeling of the viscoelastic sandwich beam.
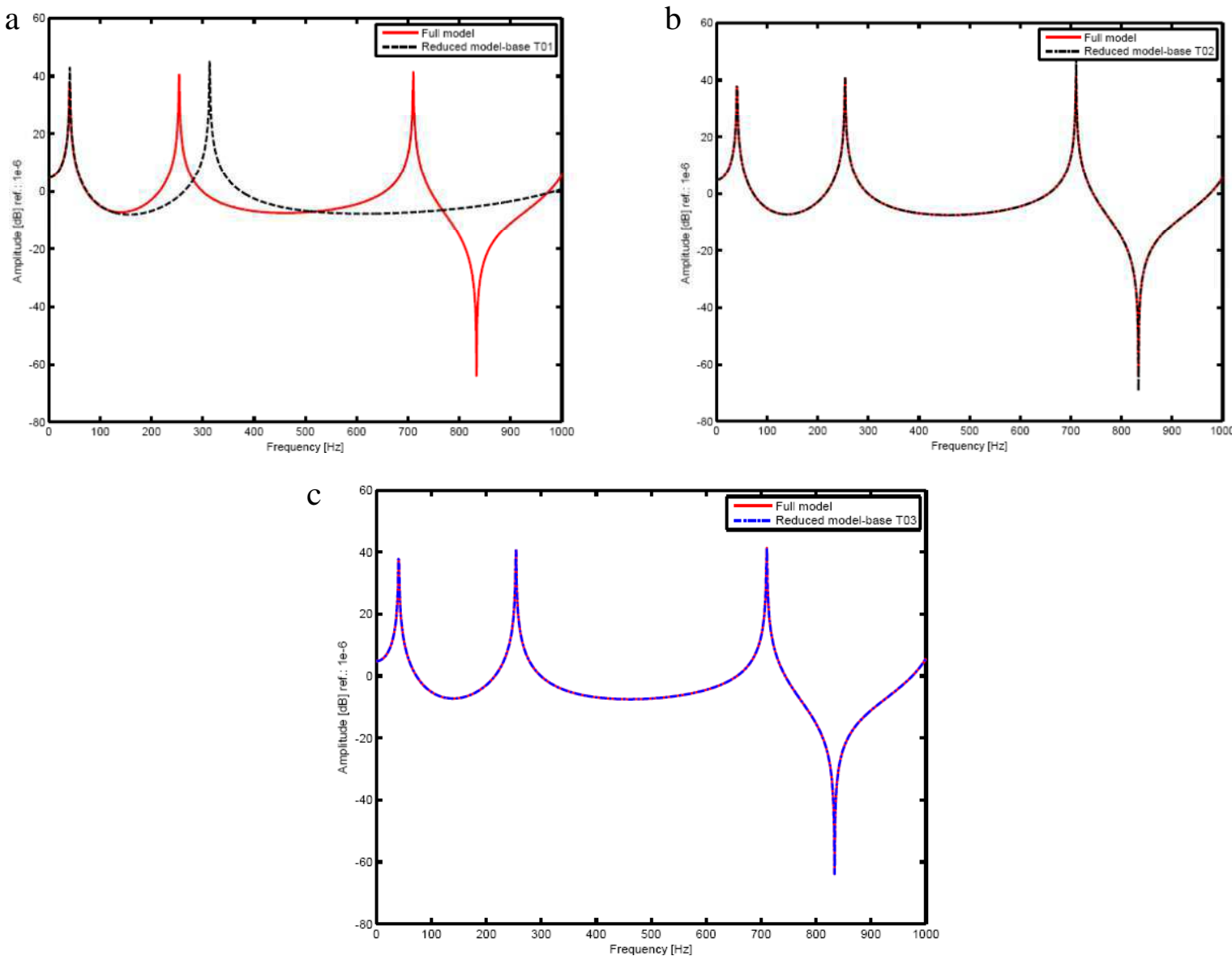

Figure4: FRFs for the full and the reduced systems by using $T_{01}(\mathrm{a}), T_{02}(\mathrm{~b}), T_{03}(\mathrm{c})$ 
The interest here is to verify the accuracy and the performance of the direct condensation methods applied to viscoelastically damped structure by comparing both Guyan static condensation and Dynamic condensation methods.

The first test is intended to evaluate the Guyan condensation method by using $T_{01}$ base which contains the static modes; $T_{01}=\left[\Theta_{0}\right](1000 \times 520)$, with $\mathrm{m}=20$ dofs and $\mathrm{s}=480$ dofs. Then, the dynamic condensation method is tested by using $T_{02}$ and $T_{03}$ bases which are obtained by enriched the static base by some slave modes. For $T_{02}=\left[\begin{array}{ll}\Theta_{0} & \phi_{s p 1}\end{array}\right](1000 \times 530)$, static Guyan transformation is enriched by $T_{s p_{1}}$ of 10 slave modes and for $T_{03}=\left[\begin{array}{ll}\Theta_{0} & \phi_{s p 2}\end{array}\right]$ $(1000 \times 570)$, the static Guyan transformation is enriched by $T_{s p_{2}}$ of 50 slave modes.

Figure 4 (a), (b) and (c) shows the frequency responses computed by using three reduction bases $T_{01} ; T_{02} ; T_{03}$ and compared to the amplitude of the response of the reference full model in the frequency band of interest [0-1000] Hz. As can be clearly seen, the accuracy is continuously improved upon successive enrichment of the reduction basis by the inclusion of 10 slave modes and 50 slave modes for $T_{02} ; T_{03}$ respectively. This leads to confirm that the use of dynamic condensation is sufficient to represent with good accuracy the dynamic behavior of viscoelastic sandwich structure.

Table 3 provides the comparison between the time computation of full model and Guyan and dynamic condensation methods using $T_{01} ; T_{02} ; T_{03}$. It shows that the use of enrichment basis $T_{02} ; T_{03}$ take a little bit more time of computing FRF than the use of static base $T_{01}$ but make a drastic reduction which up to $90 \%$ with $T_{02}$ and $87 \%$ with $T_{03}$ compared to full model.

Moreover, one can take advantage of dynamic condensation method which combines the compromise of accuracy and time performance leading to a drastic reduction for the structures incorporating viscoelastic materials.

\begin{tabular}{lcccr}
\hline Model & Full & $T_{01}$ & $T_{02}$ & $T_{03}$ \\
\hline Computing FRF (s) & 100 & 6 & 10 & 12 \\
$\%$ of reduction & - & 94 & 90 & 87
\end{tabular}

Table 3: Times evaluations of full and reduced methods.

The interest now is to compare the performance and the accuracy of the Guyan condensation method and the dynamic condensation method for the same order of both transformations. Indeed, we construct a Guyan transformation matrix with master dofs and slave dofs, having the same size with the dynamic transformation base $T_{03}$. We consider $T_{\text {Guyan }}=T_{03}(1000 \times 570)$, in the frequency band of interest [0-1200] Hz. 


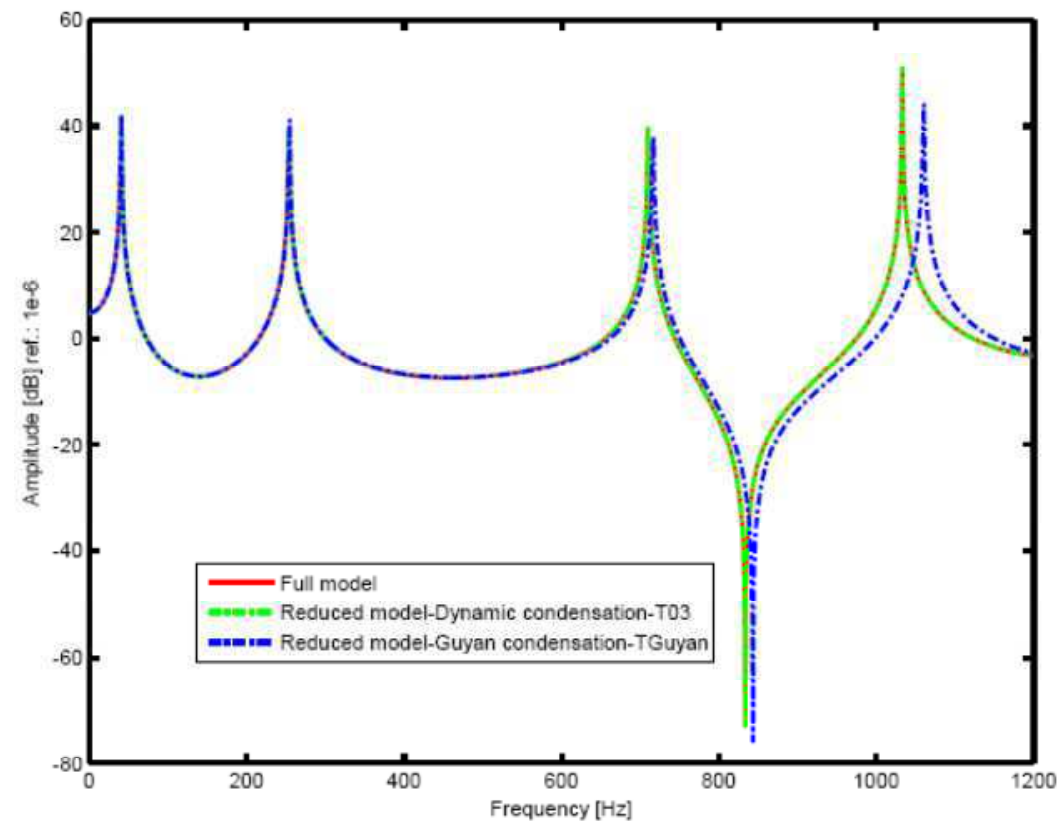

Figure5: FRFs for the full and the reduced systems-Guyan condensation/Dynamic condensation

Figure 5 shows the FRFs responses of the viscoelastic sandwich beam for the Guyan condensation method and the dynamic condensation method compared with those of full model. It can be seen that the frequency response of the dynamic condensation stick with of full model more than which is derived from Guyan condensation. The observed differences between the two direct condensation method lead to conclude that the Guyan condensation basis is not capable of overall accurately representing of the dynamic behavior of viscoelastic sandwich structures compared to the dynamic condensation method which gives satisfactory significant more accurate prediction. In fact, the static method is limited by a certain frequency called cutoff frequency which defines the domain of validity of the method and from which the results are not acceptable. For this example the cutoff frequency is around $1000 \mathrm{~Hz}$. This result confirms that the use of dynamic condensation method is more efficient to represent with good accuracy the dynamic behavior of viscoelastic sandwich structures. 


\section{CONCLUSIONS}

Finite element modeling procedures of structures incorporating viscoelastic materials are able to reproduce the FRFs responses before and after reduction was implemented, with emphasis placed on the GHM model and the implementation of two model order reduction methods: the Guyan condensation method and the dynamic condensation method.

An academic example derived from the industrial interest for the viscoelastic sandwich structures was used to illustrate the efficiency and the performance of these methods for predicting the dynamic behavior of these structures. The obtained results demonstrated the effectiveness of the two condensation strategy mainly in terms of the drastic reduction of the whole number of dofs and CPU time's computations.

\section{REFERENCES}

[1] J. Salençon, Viscoélasticité, Presse des ponts et chaussés, Paris, 1983.

[2] J.D. Ferry, Viscoelastic properties of polymers. John Wiley and Sons, New York, 1980.

[3] D.J. Mc-Tavish, P.C. Hughes, Dynamics of viscoelastic structure-a time domain, finite element formulation. ASME, Int. J. applied mechanics, 52, 897-906, 1985.

[4] D.J. Mc-Tavish, P.C. Hughes, Modeling of linear viscoelastic space structures, ASME, Int. J. vibration and acoustics of applied mechanics, 52, 103-113, 1993.

[5] R.J. Guyan, Reduction of Stiffness and Mass Matrices, AIAA Journal, Vol. 3(2), 38, 1965.

[6] C.H. Park, D.J. Inman, M.J. Lam, Model reduction of Viscoelastic finite element models. Int. J. Sound and Vibration, 219, 619-637, 1999.

[7] R. R, Jr. Craig., M.C.C.Bampton, Coupling of substructures for dynamic analyses, AIAA Journal, 6(7):1313-1319, 1968.

[8] B. Bilasse, EM. Daya, Modélisation numérique des vibrations linéaires et non linéaires des structures sandwiches à âme viscoélastique. Thèse présentée à l'université de Metz France, 2010. 